\title{
Postnatal Changes in Neonatal Acylcarnitine Profile
}

\author{
JOCHEN MEYBURG, ANDREAS SCHULZE, DIRK KOHLMUELLER, OTWIN LINDERKAMP, AND \\ ERTAN MAYATEPEK
}

Department of Neonatology [J.M., O.L.] and Division of Metabolic Diseases, Department of General Pediatrics [A.S., D.K., E.M.], University Children's Hospital, 69120 Heidelberg, Germany.

\begin{abstract}
ABST
Electrospray-tandem mass spectrometry represents a power-
ful method for detection of inborn errors of fatty acid metabo-
lism. In the present study, it was used to examine neonatal
carnitine metabolism, which reflects fatty acid metabolism. In 70
healthy neonates, blood samples were taken from the umbilical
cord and by heel-stick puncture in full-term neonates on postna-
tal d 5 . Cord blood specimens were also obtained from 15
preterm and 10 small-for-gestational-age infants. Acylcarnitine
concentrations were measured in dried blood spots by electro-
spray tandem mass spectrometry. Compared with cord blood, the
levels of nearly all acylcarnitine species were significantly higher
on the postnatal d 5, whereas free carnitine remained unchanged.
Total acylcarnitine/free carnitine-ratio increased, whereas the
free carnitine/total carnitine-ratio $(0.54 \pm 0.05 ; p<0.01)$ further
decreased. A reduced availability of free carnitine in the early
\end{abstract}
neonatal period may affect fatty acid oxidation and thus be of potential pathophysiological relevance under conditions with higher energy demands, e.g. in sepsis. Cord blood concentrations of free carnitine, total carnitine, and total acylcarnitines were strongly related to birth weight $(p<0.01)$. Lower umbilical artery $\mathrm{pH}$, i.e. mild hypoxia, caused accumulation of mainly long-chain acylcarnitines. This implicates that long-chain acylcarnitines could serve as a parameter of perinatal asphyxia. (Pediatr Res 49: 125-129, 2001)

FC, free carnitine

Abbreviations:

$\mathbf{m} / \mathbf{z}$, mass to charge ratio of ions

tAC, total acylcarnitines

TC, total carnitine
In utero, the main substrates for fetal metabolism are amino acids and glucose. The human placenta is also permeable to FFA. However, they are mainly stored as triglycerides in adipose tissues and liver because of the constant supply of glucose and amino acids and a low capacity of fetal tissues for FFA oxidation. The cutoff of the nutrient supply in the presuckling period after birth leads to a transient period of starvation. Thus, the newborn infant is entirely dependent on the mobilization of glycogen and fat stores. With the initiation of milk feeding, neonatal metabolism switches to a high-fat diet. Therefore, it is obvious that fatty acid oxidation is very important in the early postnatal period (1).

Carnitine plays an important role in oxidation of especially long-chain fatty acids. Carnitine palmitoyltransferases I and II mediate the transport of fatty acid-carnitine esters (acylcarnitines) across the mitochondrial membranes. Carnitine acyltransferases can also be found in peroxisomes (carnitine octanoyltransferase) and endoplasmatic reticulum (microsomal carnitine acyltransferase), although their function is not yet

Received November 16, 1999; accepted July 14, 2000.

Correspondence and reprint requests: Jochen Meyburg, M.D., Department of Neonatology, University Children's Hospital, Im Neuenheimer Feld 150, 69120 Heidelberg, Germany. fully understood. In the mitochondrion, carnitine removes excess acyl groups and thus regulates the concentration of free $\mathrm{CoA}$ in the mitochondrial matrix (2). Several studies have shown that the carnitine pool in the neonate is limited $(1,3,4)$. Tandem mass spectrometry allows quantitative determination of free carnitine and various carnitine esters like acylcarnitines $(5,6)$.

In the present study, we used this technique to investigate the changes in neonatal carnitine and fatty acid metabolism during the first $5 \mathrm{~d}$ after birth. Special attention was given to perinatal factors of potential pathophysiological relevance, such as umbilical artery $\mathrm{pH}$, Apgar score, gestational age, and birth weight.

\section{METHODS}

Solvents, reagents, and internal standards. High-purity grade methanol was obtained from Merck (Darmstadt, Germany). Butanolic $\mathrm{HCl}(3 \mathrm{M})$ was prepared from high-purity butanol (Merck) and $\mathrm{HCl}$-gas. Stable isotopes used as internal standards were obtained from Neo Gen Screening (Pittsburgh, PA, U.S.A.).

Patients and blood specimen collection. We studied a total of 70 healthy appropriate-for-gestational-age (AGA) neonates, 
32 girls and 38 boys. Birth weight was $3422 \pm 509 \mathrm{~g}$ (mean \pm SD) and gestational age was $39.4 \pm 1.3$ wk. Thirteen infants were delivered by cesarean section. None of the neonates had a 1-min Apgar score lower than 6,10-min Apgar scores were 9 or 10 in all subjects. To test for a possible influence of birth weight or gestational age, 10 small-for-gestational-age (SGA) infants (birth weight below third centile: $2502 \pm 507 \mathrm{~g}$, gestational age $39.4 \pm 0.7 \mathrm{wk}$ ) and 15 preterm AGA infants (birthweight $1166 \pm 329 \mathrm{~g}$, gestational age $28.8 \pm 2.2 \mathrm{wk}$ ) were also included in the study. Blood was taken immediately after birth by puncture of the umbilical artery. Two drops of blood were spotted on a Guthrie card. A second blood sample was obtained from the term AGA infants on the postnatal d 5 by heel-stick puncture during routine neonatal metabolic screening. On d 5, breast-feeding was well established in these children with a mean daily intake of $120 \mathrm{~mL} / \mathrm{kg}$ body weight (mean fat intake $5.4 \mathrm{~g} / \mathrm{kg}$, mean carnitine supply $0.9 \mu \mathrm{mol} / \mathrm{kg}$ ) (7). Heel-stick puncture was performed 30 min after feeding. Informed consent was obtained from all mothers. The study was approved by the Ethical Committee of the University Children's Hospital, Department of Neonatology.

Sample preparation. All dried blood spots were investigated by means of electrospray-tandem mass spectrometry by the method previously described in detail $(5,6)$. Briefly, one 3-mm-diameter dot per sample was punched out from a 10$\mathrm{mm}$-diameter dried blood spot into a vial of a 96-well microtiter plate. A methanol stock solution of internal standards was prepared, containing $0.76 \mu \mathrm{M}\left[{ }^{2} \mathrm{H}_{9}\right]$ carnitine $(\mathrm{m} / \mathrm{z} 227,3), 0.19$ $\mu \mathrm{M}\left[{ }^{2} \mathrm{H}_{3}\right]$ acetylcarnitine $(\mathrm{m} / \mathrm{z} 263,4), 0.04 \mu \mathrm{M}$ each of $\left[{ }^{2} \mathrm{H}_{3}\right]$ propionylcarnitine $(\mathrm{m} / \mathrm{z} 277,4),\left[{ }^{2} \mathrm{H}_{3}\right]$ butyrylcarnitine $\left(\mathrm{m} / \mathrm{z}\right.$ 291,4), $\left[{ }^{2} \mathrm{H}_{9}\right]$ isovalerylcarnitine $(\mathrm{m} / \mathrm{z} \quad 311,4)$, $\left[{ }^{2} \mathrm{H}_{3}\right]$ octanoylcarnitine $(\mathrm{m} / \mathrm{z} 347,5),\left[{ }^{2} \mathrm{H}_{9}\right]$ myristoylcarnitine $(\mathrm{m} / \mathrm{z} 437,6)$, and $0.08 \mu \mathrm{M}\left[{ }^{2} \mathrm{H}_{3}\right]$ palmitoylcarnitine $(\mathrm{m} / \mathrm{z} 459,6)$. The samples were allowed to elute in $100 \mu \mathrm{L}$ of the stock solution for $20 \mathrm{~min}$ before the blood dots were removed. They were evaporated to dryness in a freeze dryer and then $60 \mu \mathrm{L}$ of $3 \mathrm{n} \mathrm{HCl}$ in butanol were added. The microtiter plates were sealed and incubated at $65^{\circ} \mathrm{C}$ in a forced air oven for $15 \mathrm{~min}$. After removal of the seal, excess HCl-butanol was evaporated to dryness in a freeze dryer. The derived samples were reconstituted with $100 \mu \mathrm{L}$ acetonitril/water (1:1), containing $0.025 \%$ formic acid. The internal standard was used for quantification of acylcarnitines by forming the ratio of the signals. When the specific stable isotope was not available, the following ratios were used for calculation: $\mathrm{C} 2 / \mathrm{C} 3-\mathrm{d} 3, \mathrm{C} 5: 1 / \mathrm{C} 5-\mathrm{d} 9, \mathrm{C} 6 / \mathrm{C} 5-\mathrm{d} 9$, C8:1/C8-d3, C10/C8-d3, C10:1/C8-d3, C12/C14-d9, C14:1/ C14-d9, C14OH/C14-d9, C16:1/C16-d3, C16OH/C16-d3, C16:1OH/C16-d3, C18/C16-d3， C18:1/C16-d3, $\mathrm{C} 18: 1 \mathrm{OH} / \mathrm{C} 16-\mathrm{d} 3$

Mass spectrometry. A PE Sciex API 365 triple quadrupole tandem mass spectrometer (PE Sciex, Concord, Ontario, Canada) was used with an ion spray source. Twenty-five microliters of the samples were introduced by using a PE 200 HPLCpump $(40 \mu \mathrm{L} / \mathrm{min}$ acetonitril/water $(1: 1 \mathrm{vol} / \mathrm{vol})$ containing $0.025 \%$ formic acid) and a PE 200 autosampler. The first analyzer was set to scan all ions between $\mathrm{m} / \mathrm{z} 200$ and 500 . Acylcarnitines were detected by looking for the precursor ions of $\mathrm{m} / \mathrm{z}=85$. The acylcarnitine butylester passes analyzer 1 , collides with nitrogen (fragmentation), and one of the typical fragments $(\mathrm{m} / \mathrm{z}=85)$ passes analyzer 2 , which is fixed to $\mathrm{m} / \mathrm{z}$ $=85$. It is registered by the detector while the $\mathrm{m} / \mathrm{z}$ that has passed analyzer 1 is recorded.

To enable comparisons to reported values, acylcarnitine concentrations were summarized to obtain levels for short(acetyl-, propionyl-, butyryl-, and isovalerylcarnitine), medium- (hexanoyl-, octanoyl-, octenoyl-, decanoyl-, decenoyl-, and lauroylcarnitine) and long-chain acylcarnitines (myristoyl-, myristoleyl-, hydroxymyristoyl-, palmitoyl-, hydroxypalmitoyl-, palmitoleyl-, hydroxypalmitoleyl-, stearoyl-, hydroxystearoyl-, oleyl-, and hydroxyoleylcarnitine). Short-, medium- and long-chain acylcarnitines were again summarized to yield total acylcarnitine concentrations. Cord blood and postnatally drawn blood samples were compared with the $t$ test for paired samples. Cord blood samples of the AGA, SGA, and preterm infants were tested for differences with the $t$ test for unpaired samples. Correlations with perinatal factors were calculated using the Pearson correlation. Differences with $p<$ 0.05 were regarded statistically significant.

\section{RESULTS}

The levels of FC and acylcarnitines in cord blood and in the postnatal blood samples are summarized in Table 1. No differences in the levels of FC were noted between cord and 5-d blood. The concentrations of carnitine esters with saturated acyl chains, however, were found to be considerably higher on the postnatal d 5. Similar results were obtained for acylcarnitines with monounsaturated acyl chains. Hydroxylated longchain acylcarnitines remained unchanged during the first $5 \mathrm{~d}$. The ratio of tAC to FC increased significantly on $\mathrm{d} 5$. On the other hand, the ratio of FC to TC decreased.

In the cord blood samples, concentrations of TC, FC, tAC, short-chain (all $p<0.01$ ), and long-chain $(p<0.05)$ acylcarnitines increased with increasing birth weight (Fig. 1). Significant positive correlations were noted for acetyl- $(p=0.01)$, propionyl- $(p<0.001)$, butyryl- $(p<0.05)$, isovaleryl- $(p<$ $0.05)$, oleyl- $(p<0.05)$, and hydroxyoleylcarnitine $(p<0.05)$.

In cord blood, concentrations of acetyl- $(p<0.01)$, propionyl- $(p<0.05)$, myristoyl- $(p<0.01)$, myristoleyl- $(p<0.01)$, palmitoyl- $(p<0.05)$, stearoyl- $(p<0.05)$, and oleylcarnitine $(p<0.05)$ increased with decreasing umbilical arterial $\mathrm{pH}$ (Fig. 2) and 5-min Apgar score.

The influence of birth weight on the concentrations of FC and short-chain acylcarnitines was still present in the postnatal blood samples drawn on d 5. Correlations were found for FC $(p<0.01)$, acetyl- $(p<0.01)$ and propionylcarnitine $(p<$ $0.01)$. On the postnatal d 5, FC and acylcarnitine levels were not related to gestational age or umbilical artery $\mathrm{pH}$. Carnitine levels did not correlate with gestational age, neither at birth nor on $\mathrm{d} 5$. No differences were noted regarding the concentrations of FC and acylcarnitines between girls and boys or between normal labor and delivery by cesarean section.

Cord blood levels of free carnitine, total carnitine, and total, short-, medium-, and long-chain acylcarnitine were lower in preterm compared with term infants (Fig. 3). Significantly lower values were also found for propionyl- $(p=0.001)$, 
Table 1. Concentrations of FC and acylcarnitines in cord blood and postnatally drawn blood samples (mean $\pm S D)$

\begin{tabular}{|c|c|c|c|}
\hline & $\begin{array}{l}\text { Cord blood } \\
{[\mu \mathrm{mol} / \mathrm{L}]^{*}}\end{array}$ & $\begin{array}{c}\text { Day } 5 \text { after } \\
\text { birth } \\
{[\mu \mathrm{mol} / \mathrm{L}]^{*}}\end{array}$ & $p$-Value \\
\hline $\mathrm{TC}$ & $51.5 \pm 14.4$ & $55.7 \pm 16.0$ & $<0.01$ \\
\hline $\mathrm{FC}$ & $31.6 \pm 10.2$ & $30.6 \pm 10.6$ & NS \\
\hline tAC & $19.9 \pm 4.8$ & $25.1 \pm 6.4$ & $<0.001$ \\
\hline Short-chain acylcarnitines & $13.1 \pm 3.5$ & $15.5 \pm 4.6$ & $<0.001$ \\
\hline Medium-chain acylcarnitines & $0.8 \pm 0.2$ & $0.9 \pm 0.2$ & $<0.001$ \\
\hline Long-chain acylcarnitines & $6.0 \pm 1.3$ & $8.6 \pm 2.1$ & $<0.001$ \\
\hline $\mathrm{tAC} / \mathrm{FC}$ & $0.65 \pm 0.12$ & $0.86 \pm 0.17$ & $<0.01$ \\
\hline $\mathrm{FC} / \mathrm{TC}$ & $0.61 \pm 0.04$ & $0.54 \pm 0.05$ & $<0.01$ \\
\hline \multicolumn{4}{|l|}{ Short-chain acylcarnitines } \\
\hline Acetylcarnitine (C2) & $10.3 \pm 3.1$ & $12.6 \pm 4.1$ & $<0.001$ \\
\hline Propionylcarnitine (C3) & $2.0 \pm 0.6$ & $2.2 \pm 0.7$ & $<0.05$ \\
\hline Butyrylcarnitine (C4) & $0.41 \pm 0.13$ & $0.47 \pm 0.13$ & NS \\
\hline Isovalerylcarnitine (C5) & $0.24 \pm 0.09$ & $0.27 \pm 0.08$ & $<0.05$ \\
\hline Isovalerylcarnitine (C5:1) & $0.13 \pm 0.05$ & $0.13 \pm 0.05$ & NS \\
\hline \multicolumn{4}{|l|}{ Medium-chain acylcarnitines } \\
\hline Hexanoylcarnitine (C6) & $0.21 \pm 0.06$ & $0.23 \pm 0.06$ & NS \\
\hline Octanoylcarnitine (C8) & $0.12 \pm 0.06$ & $0.14 \pm 0.06$ & $<<0.05$ \\
\hline Octenoylcarnitine (C8:1) & $0.13 \pm 0.06$ & $0.17 \pm 0.08$ & $<0.01$ \\
\hline Decanoylcarnitine (C10) & $0.10 \pm 0.05$ & $0.12 \pm 0.05$ & $<0.05$ \\
\hline Cecenoylcarnitine (C10:1) & $0.10 \pm 0.04$ & $0.11 \pm 0.04$ & NS \\
\hline Lauroylcarnitine (C12) & $0.15 \pm 0.06$ & $0.18 \pm 0.09$ & $<0.05$ \\
\hline \multicolumn{4}{|l|}{ Long-chain acylcarnitines } \\
\hline Myristoylcarnitine (C14) & $0.23 \pm 0.09$ & $0.30 \pm 0.11$ & $<0.001$ \\
\hline Myristoleylcarnitine (C14:1) & $0.12 \pm 0.05$ & $0.13 \pm 0.06$ & NS \\
\hline Palmitoylcarnitine (C16) & $2.6 \pm 0.8$ & $3.8 \pm 1.3$ & $<0.001$ \\
\hline Palmitoleylcarnitine (C16:1) & $0.17 \pm 0.06$ & $0.29 \pm 0.11$ & $<0.001$ \\
\hline Stearoylcarnitine (C18) & $1.3 \pm 0.4$ & $1.6 \pm 0.5$ & $<0.001$ \\
\hline Oleylcarnitine (C18:1) & $1.1 \pm 0.4$ & $1.9 \pm 0.6$ & $<0.001$ \\
\hline Hydroxymyristoylcarnitine $(\mathrm{C} 14 \mathrm{OH})$ & $0.13 \pm 0.06$ & $0.13 \pm 0.05$ & NS \\
\hline Hydroxypalmitoylcarnitine $(\mathrm{C} 16 \mathrm{OH})$ & $0.14 \pm 0.07$ & $0.14 \pm 0.06$ & NS \\
\hline $\begin{array}{l}\text { Hydroxypalmitoleylcarnitine } \\
(\mathrm{C} 16: 1 \mathrm{OH})\end{array}$ & $0.14 \pm 0.07$ & $0.16 \pm 0.08$ & NS \\
\hline Hydroxyoleylcarnitine $(\mathrm{C} 18: 1 \mathrm{OH})$ & $0.07 \pm 0.04$ & $0.08 \pm 0.04$ & NS \\
\hline
\end{tabular}

* Except tAC/FC and $\mathrm{FC} / \mathrm{TC}$ ratios.

lauroyl- $(p=0.002)$, myristoleyl- $(p=0.001)$, hydroxymyristoyl- $(p<0.05)$, palmitoyl- $(p<0.001)$, palmitoleyl- $(p<$ $0.05)$, hydroxypalmitoyl- $(p<0.05)$, stearoyl- $(p<0.01)$, and oleylcarnitine $(p<0.001)$. Carnitine levels in the cord blood of SGA infants were lower than in term AGA children (Fig. 3). However, the differences were not statistically significant. Compared with the SGA infants, the cord blood levels of the preterm infants tended to be lower.

\section{DISCUSSION}

Maternal plasma carnitine concentrations are significantly lower from wk 12 of gestation, compared with nonpregnant women. These differences become more pronounced toward the end of the pregnancy. Levels of FC decrease to a greater extent than acylcarnitine concentrations (8). Maternal plasma carnitine concentrations have been reported to be lower than in umbilical cord blood, implicating active carnitine transport across the placenta $(9,10)$. However, no differences between maternal and cord blood have been detected in another study (11).

Only a few studies on carnitine status in cord blood have been carried out before $(4,12,13)$. In all these studies, plasma

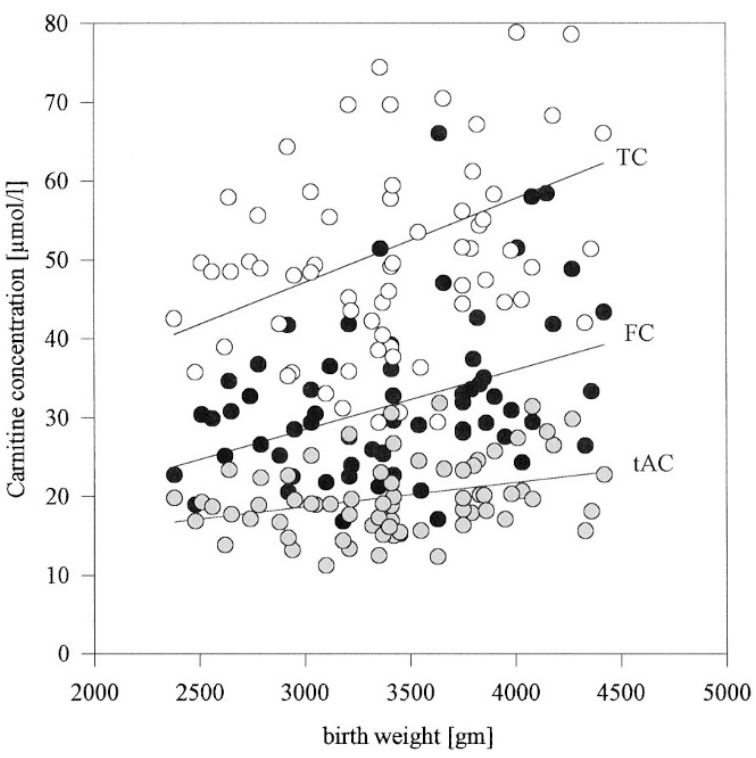

Figure 1. Correlation of FC, TC, and tAC with birth weight in cord blood of AGA infants.

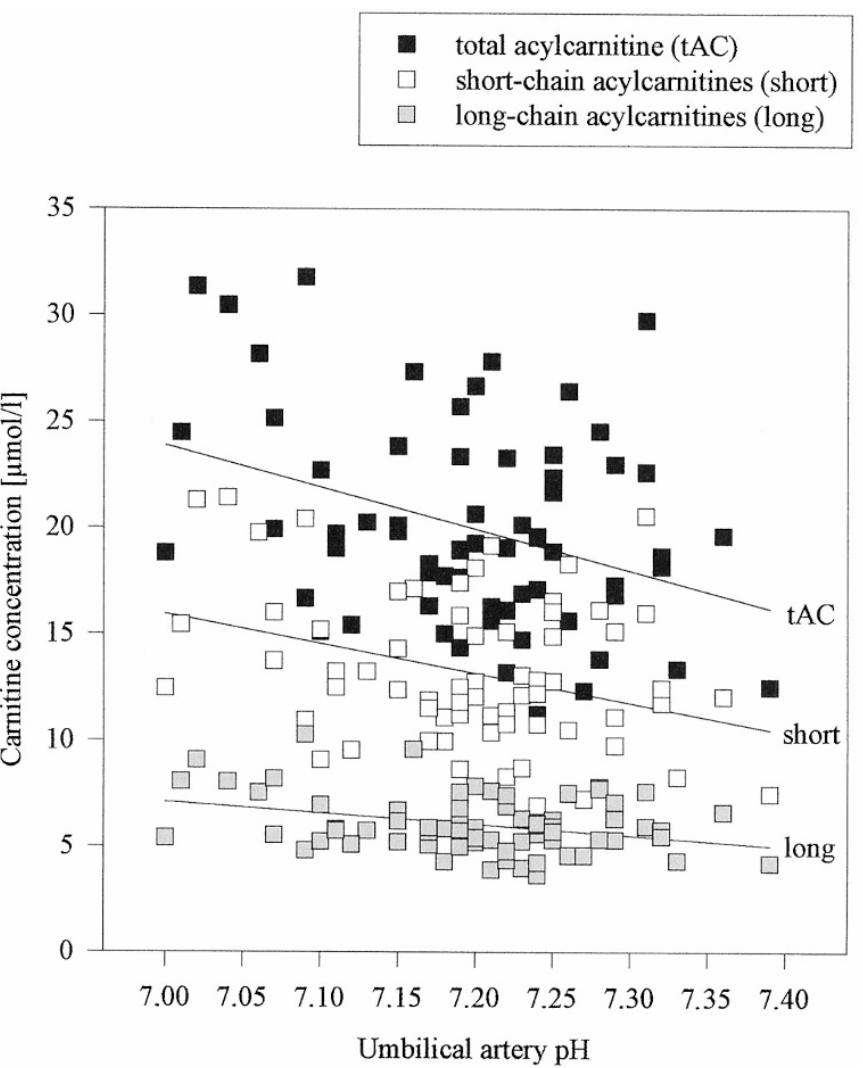

Figure 2. Correlation of tAC and short- and long-chain acylcarnitine with umbilical artery $\mathrm{pH}$ in cord blood of AGA infants.

carnitine levels were measured. Because the intraerythrocytic carnitine pool can comprise to $73 \%$ of whole blood carnitine content (4), investigations on whole blood by tandem mass 
AGA term infants SGA term infants Preterm infants

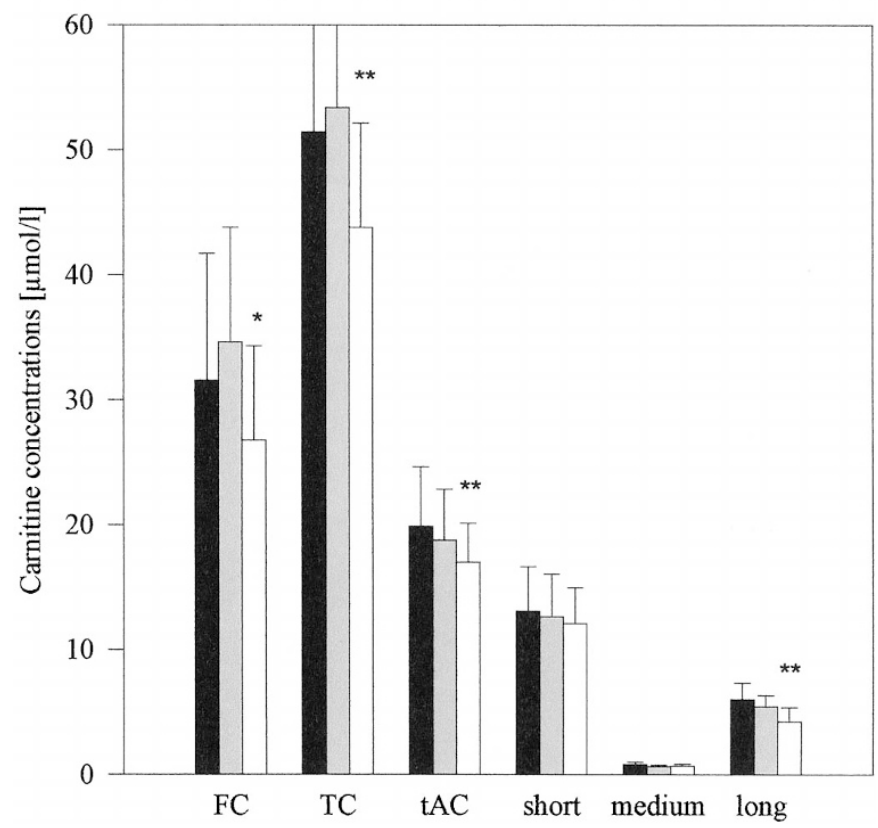

Figure 3. Cord blood levels of FC, TC, tAC, and short-, medium-, and long-chain acylcarnitines in AGA, SGA, and preterm infants $\left({ }^{*} p<0.05\right.$; $* * p<0.01$ preterm compared with AGA term infants).

spectrometry may be more appropriate. In whole blood samples of 14 full-term infants, we found markedly higher levels of total $(47.8 \pm 15.1$ versus $37.8 \pm 9.7 \mu \mathrm{mol} / \mathrm{L})$ and long-chain acylcarnitines $(7.1 \pm 2.3$ versus $1.8 \pm 0.5 \mu \mathrm{mol} / \mathrm{L})$ compared with plasma. Because of these differences, carnitine levels obtained by tandem mass spectrometry cannot be easily compared with previous data.

The main function of carnitine is the transport of fatty acids across the mitochondrial membranes. In the liver carnitine is only needed for the transport of long-chain fatty acids, whereas in other tissue like skeletal muscle or myocardium, carnitine is also important for the transport of short- and medium-chain fatty acids (2). The intramitochondrial relationship between acyl-CoA and free CoA is reflected by the extramitochondrial tAC to FC ratio. Thus, high plasma levels of acylcarnitines reflect reduced bioavailability of acyl-CoA in the mitochondrion. The $\mathrm{tAC} / \mathrm{FC}$ has therefore been called a marker of "carnitine insufficiency" $(14,15)$. On the other hand, the ratio of FC to TC is a useful parameter to assess the availability of FC. Because higher acylcarnitine levels in whole blood are compensated for by a tendency to overestimate FC concentrations using tandem mass spectrometry (16), the ratios obtained by different techniques should be comparable. In fact, our tandem mass spectrometry data corresponds well to published data for cord blood $(12,13)$ and neonates (17).

Longitudinal observations of carnitine metabolism within the neonatal period and early infancy are rare. Plasma levels of TC, FC, and tAC increase after $1-4$ wk after birth $(9,17)$ and are significantly higher in older children when compared with neonates (18). During infancy, tAC/FC decreases to 0.4 whereas FC/TC increases to 0.7 (15). The highest tAC/FC and lowest FC/TC can thus be found within the neonatal period. Our results support this observation in detail. Within the first 5 postnatal d, levels of all acylcarnitines increased, whereas the concentration of FC remained unchanged (Table 1). Decreased availability of free carnitine within the first postnatal days may impair fatty acid oxidation. For the newborn, this may be potentially harmful in conditions with higher energy demand, e.g. during septicemia.

Levels of TC, FC, and tAC were significantly related to birth weight. Lower values were found in the SGA group compared with the AGA infants. Rising carnitine levels with higher birth weights may reflect increasing tissue carnitine stores, which are known to be strongly related to body weight and muscle mass $(3,19)$. In our study on neonates with $37-42$ wk of gestation, no significant influence of gestational age could be detected. Nevertheless, cord blood levels of preterm children were significantly lower than those of full-term infants. Reported cord blood plasma levels of TC, FC, and tAC are higher in preterm compared with term infants $(4,12,13,20)$.

Acylcarnitine concentrations were inversely correlated with umbilical artery $\mathrm{pH}$ and 5-min Apgar score. This suggests that mild acidemia and hypoxia leads to accumulation of short- and long-chain acylcarnitines. Accumulation of long-chain acylcarnitines is known as one of the main causes of arrhythmias and cellular damage in ischemic myocardium $(21,22)$. This has also been shown for neonatal rat myocytes (23). In six infants with severe perinatal asphyxia $(\mathrm{pH}<7.0$, base excess $<-20)$ we found significantly higher levels of total $(26.7 \pm 4.9$ versus $19.9 \pm 4.8 \mu \mathrm{mol} / \mathrm{L})$ and long-chain acylcarnitines $(7.8 \pm 1.5$ versus $5.9 \pm 1.4 \mu \mathrm{mol} / \mathrm{L}$ ) immediately after birth compared with cord blood of healthy term infants. We therefore speculate that levels of long-chain acylcarnitines could serve as a useful parameter for perinatal asphyxia.

In summary, we present representative levels of carnitine and its esters as determined by electrospray tandem mass spectrometry in whole blood specimen within the early neonatal period. Acylcarnitines increase after birth while free carnitine remains unchanged. In full-term infants, carnitine concentrations depend strongly on birth weight, and further studies on the influence of gestational age are needed. Mild acidosis and hypoxia lead to accumulation of long-chain acylcarnitines. The availability of free carnitine, given by the tAC/FC and FC/TC ratios, is lower compared with cord blood and older children. This restriction of fatty acid oxidation capacity could be of potential pathophysiological relevance during the first days of life, especially under conditions with higher energy demands.

\section{REFERENCES}

1. Hahn P, Novak M 1985 How important are carnitine and ketones for the newborn infant? Fed Proc 44:2369-2373

2. Ramsay RR 1994 Carnitine and its role in acyl group metabolism. Essays Biochem 28:47-61

3. Shenai JP, Borum PR 1984 Tissue carnitine reserves of newborn infants. Pediatr Res 18:679-682

4. Shenai JP, Borum PR, Mohan P, Donlevy SC 1983 Carnitine status at birth of newborn infants of varying gestation. Pediatr Res 17:579-582

5. Chace DH, Hillman SL, Van HJ, Naylor EW 1997 Rapid diagnosis of MCAD deficiency: quantitatively analysis of octanoylcarnitine and other acylcarnitines in newborn blood spots by tandem mass spectrometry. Clin Chem 43:2106-2113 
6. Rashed MS, Ozand PT, Bucknall MP, Little D 1995 Diagnosis of inborn errors of metabolism from blood spots by acylcarnitines and amino acids profiling using automated electrospray tandem mass spectrometry. Pediatr Res 38:324-331

7. Penn D, Dolderer M, Schmidt-Sommerfeld E 1987 Carnitine concentrations in the milk of different species and infant formulas. Biol Neonate 52:70-79

8. Schoderbeck M, Auer B, Legenstein E, Genger H, Sevelda P, Salzer H, Marz R, Lohninger A 1995 Pregnancy-related changes of carnitine and acylcarnitine concentrations of plasma and erythrocytes. J Perinat Med 23:477-485

9. Novak M, Monkus EF, Chung D, Buch M 1981 Carnitine in the perinatal metabolism of lipids. I. Relationship between maternal and fetal plasma levels of carnitine and acylcarnitines. Pediatrics 67:95-100

10. Bargen-Lockner C, Hahn P, Wittmann B 1981 Plasma carnitine in pregnancy. Am J Obstet Gynecol 140:412-414

11. Schmidt-Sommerfeld E, Penn D, Wolf H 1981 The influence of maternal fat metabolism on fetal carnitine levels. Early Hum Dev 5:233-242

12. Giannacopoulou C, Evangeliou A, Matalliotakis I, Relakis K, Sbirakis N, Hatzidaki E, Koumandakis E 1998 Effects of gestation age and of birth weight in the concentration of carnitine in the umbilical plasma. Clin Exp Obstet Gynecol 25:42-45

13. Bernardini I, Evans MI, Nicolaides KH, Economides DL, Gahl WA 1991 The feta concentrating index as a gestational age-independent measure of placental dysfunction in intrauterine growth retardation. Am J Obstet Gynecol 164:1481-1487

14. Böhles HJ, Evangeliou A, Bervoets K, Sewell AC 1994 Carnitine esters in metabolic disease. J Pediatr 153:S57-S61
15. Winter SC, Zorn EM, Hugh Vance W 1990 Carnitine deficiency. Lancet 335:981-982 16. Johnson DW 1999 Inaccurate measurement of free carnitine by the electrospray tandem mass spectrometry screening method for blood spots. J Inherit Metab Dis 22:201-202

17. Campoy C, Bayes R, Peinado JM, Rivero M, Lopez C, Molina-Font JA 1998 Evaluation of carnitine nutritional status in fullterm newborn infants. Early Hum Dev 53:S149-S164

18. De Sousa C, English NR, Stacey TE, Chalmers RA 1990 Measurement of L-carnitine and acylcarnitines in body fluids and tissues in children and adults. Clin Chim Acta 187:317-328

19. Penn D, Schmidt-Sommerfeld E, Pascu F 1981 Decreased tissue carnitine concentrations in newborn infants receiving total parenteral nutrition. J Pediatr 98:976-978

20. Sachan DS, Smith RB, Plattsmier J, Lorch V 1989 Maternal, cord, and neonatal carnitine correlations and lipid profiles of various birthweight infants. Am J Perinatol 6:14-17

21. Yamada KA, McHowat J, Yan GX, Donahue K, Peirick J, Kleber AG, Corr PB 1994 Cellular uncoupling induced by accumulation of long-chain acylcarnitine during ischemia. Circ Res 74:83-95

22. Hütter JF, Soboll S 1992 Role of fatty acid metabolites in the development of myocardial ischemic damage. Int J Biochem 24:399-403

23. Knabb MT, Saffitz JE, Corr PB, Sobel BE 1986 The dependence of electrophysiological derangements on accumulation of endogenous long-chain acyl carnitine in hypoxic neonatal rat myocytes. Circ Res 58:230-240 